\title{
Parameters of the network
}

\begin{tabular}{clc}
\hline \hline Symbol & Meaning & Default value \\
\hline$N$ & number of Potts units & 1000 \\
$S$ & number of states per unit & 7 \\
$p$ & number of stored LTM patterns & 200 \\
$a$ & sparsity of patterns & 0.25 \\
$c_{\mathrm{m}}$ & number of presynaptic units per unit & 150 \\
$U$ & threshold common to all units & 0.1 \\
$\beta$ & effective inverse temperature & 11 \\
$\tau_{1}$ & timescale for "fields" (rk $)$ & 10 \\
$\tau_{2}$ & timescale for adaptive thresholds $\left(\theta_{i}^{k}\right)$ & 200 \\
$\tau_{\mathrm{A}}$ & timescale for fast inhibition $\left(\theta_{i}^{A}\right)$ & 5 \\
$\tau_{\mathrm{B}}$ & timescale for slow inhibition $\left(\theta_{i}^{B}\right)$ & 100000 \\
$\gamma_{\mathrm{A}}$ & proportion of fast inhibition & 0.5 \\
$w$ & self-reinforcement parameter & 0.8 \\
$L$ & number of patterns in STM & 16 \\
$\Delta \theta$ & the amount of decrease in adaptive threshold & 0.3 \\
$\lambda$ & strength of heteroassociative connections & 0.1 \\
\hline
\end{tabular}

